\title{
ARBORIZAÇÃO DOS BAIRROS ALTO BRANCO, LAURITZEN E SANTO ANTÔNIO, CAMPINA GRANDE/PB: UM ESTUDO COMPARATIVO
}

\author{
Ivan Coelho Dantas ${ }^{1}$, Thiago Pereira Chaves $^{2}$, Delcio de Castro Felismino ${ }^{3}$;Vânia Maria \\ Gomes Ferreira ${ }^{4}$
}

(recebido em 06.11.2010 e aceito para publicação em 08.07.2011)

\section{RESUMO}

Devido à falta de planejamento e de consciência ambiental por parte da população, a arborização urbana não tem recebido a importância devida, o que acarreta uma série de danos causados pelas árvores, as quais, quase sempre, são retiradas ou substituídas. Este trabalho teve como objetivo realizar um inventário quali-quantitativo das espécies existentes na arborização em ruas, avenidas e praças dos bairros do Alto Branco, Lauritzen e Santo Antônio da cidade de Campina Grande - PB, e comparar os resultados com os dos inventários realizados em 1998 e em 2000. A coleta de dados foi realizada através de visitas "in loco" percorrendo ruas, avenidas e praças, sendo inventariadas todas as árvores a partir de 1,5 metros. Foram catalogadas 1.698 árvores no bairro do Alto Branco, 771 no Lauritzen e 559 no Santo Antônio, representando, respectivamente, um aumento de 97,9\%, 64,0\%, e $36,1 \%$ no número de espécimes com relação ao inventário de 1998. A espécie de maior destaque foi Ficus benjamina L. com aumento acentuado de 2.215,8\%, em número de espécimes plantados, principalmente em calçadas, mesmo sendo inadequados para arborização das mesmas.

Palavras-chave: Inventário; Bairros de Campina Grande; Impacto ambiental.

\footnotetext{
${ }^{1}$ Farmacêutico, Departamento de Biologia da Universidade Estadual da Paraíba - UEPB, Campina Grande. ivancd@gmail.com

${ }^{2}$ Biólogo, Mestrando em Ciência e Tecnologia Ambiental pela Universidade Estadual da Paraíba UEPB. Campina Grande. pereira_thiago@msn.com

${ }^{3}$ Biólogo, Departamento de Biologia da Universidade Estadual da Paraíba - UEPB, Campina Grande. delciofelismino@gmail.com

${ }^{4}$ Licenciatura Plena em Letras/Inglês. vaninhamgf@hotmail.com
}

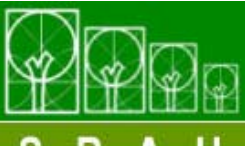

$\mathbf{S} \cdot \mathbf{B} \cdot \mathbf{A} \cdot \mathbf{U}$ Soc. Bras. de Arborização Urbana

REVSBAU, Piracicaba - SP, v.6, n.2, p.76-89, 2011 


\section{URBAN FOREST OF ALTO BRANCO, LAURITZEN AND SANTO ANTÔNIO NEIGHBORHOODS IN CAMPINA GRANDE, PB: A COMPARATIVE STUDY}

\section{ABSTRACT}

Due to the lack of planning and environmental consciousness from population, urban forest has not been treated with correct importance, can the trees cause damages, because of this they are almost always cut down or substituted. This study aimed realize a quali-quantitative inventory of existent species in urban forest of streets, avenues and squares of Alto Branco, Lauritzen and Santo Antônio neighborhoods in Campina Grande, PB, as well as compare it with the results of inventories carried in 1998 and 2000. The data collection process was applied through "in loco" visits walking on streets, avenues and squares. Were inventoried every tree from 1.5 meters tall. Were cataloged 1,698 trees in Alto Branco neighborhood, 771 in Lauritzen and 559 in Santo Antônio, representing respectively, a raise of 97.9\%, $64.0 \%$, and $36.1 \%$ on specimens number, comparing to 1998 inventory. The most spotlighted species was Ficus benjamina L. which had a marked increase of $2,215.8 \%$ in number of planted specimens, mainly in sidewalks, although it is inadequate for this purpose.

Keywords: Inventory; Campina Grande neighborhoods; Environmental impact.

\section{INTRODUÇÃO}

Entende-se por arborização urbana toda cobertura vegetal de porte arbóreo existente nas cidades. Esta ocupa, basicamente, áreas livres de uso público e potencialmente coletivas; áreas livres particulares; e acompanhando o sistema viário.

A arborização urbana, segundo Balensiefer e Wiecheteck (1987) e Souza (2001), constitui um elemento de grande importância para a elevação da qualidade de vida da população, seja em grandes centros urbanos quanto em pequenas cidades. Com suas características, a arborização urbana é capaz de contribuir para amenizar os efeitos da alta temperatura nas cidades e propiciar um ambiente visualmente agradável aos habitantes e visitantes.

No entanto, a arborização necessita, como qualquer outra atividade, de profissionais bem capacitados para a sua realização. Como relatam Christo e Dias (2006) a arborização (4) (P)

$\mathbf{S} \cdot \mathbf{B} \cdot \mathbf{A} \cdot \mathbf{U}$ Soc. Bras. de Arborização Urbana

REVSBAU, Piracicaba - SP, v.6, n.2, p.76-89, 2011 
de vias, quando mal planejada, pode acarretar dificuldade de circulação para as pessoas, entupimento de encanamentos pluviais, quebra de calçadas e até mesmo o desmonte de muros. Além dessa multiplicidade de fatores relativos ao ambiente artificial criado pelo homem, atualmente, o que se tem observado é o modismo e normalmente as plantas que são sugeridas como ideais e adequadas são exóticas, recomendadas por viveiristas interessados no comércio.

Para que a presença da árvore na via pública não seja inconveniente, há necessidade da escolha das espécies mais aconselhadas para cada rua ou para cada cidade (PEDROSA, 1983). De acordo com Dantas et al., (2010), a avaliação da arborização de uma cidade inicia-se com a realização de inventários ou levantamentos arbóreos que, de acordo com Rocha et al., (2004) e Melo et al., (2007) consistem em uma importante ferramenta para a fundamentação do planejamento e manejo adequado das árvores, bem como instituição de ações prioritárias.

Com base no exposto, o objetivo deste trabalho foi realizar um inventário das espécies existentes na arborização em ruas, avenidas e praças dos bairros Alto Branco, Lauritzen e Santo Antônio da cidade de Campina Grande/PB, e comparar esses dados com o inventário realizado nos anos de 1998 e 2000.

\section{MATERIAIS E MÉTODOS}

Esse trabalho foi realizado na cidade de Campina Grande - PB, que é considerada a segunda cidade mais populosa do Estado da Paraíba, possui uma população estimada em 385.276 habitantes (IBGE, 2010), com área territorial de $621 \mathrm{~km}^{2}$, sendo $96 \mathrm{~km}^{2}$ de área urbana, estando localizada a $120 \mathrm{~km}$ do litoral paraibano, (Figura 1) tendo temperatura média anual em torno dos $22^{\circ} \mathrm{C}$, podendo atingir $30^{\circ} \mathrm{C}$ nos dias mais quentes e $15^{\circ} \mathrm{C}$ nas noites mais frias do ano, com umidade relativa do ar entre 75 a $83 \%$ (MASCARENHAS, 2005).

O inventário arbóreo foi realizado através de visitas in loco percorrendo ruas, avenidas e praça, dos bairros Alto Branco, Lauritzen e Santo Antônio, utilizando o mapa urbano da divisão de bairros de acordo com a Lei 1.542/87 com o traçado das ruas, onde se observou o limite de fronteiras dos bairros. 
Foram inventariadas todas as árvores a partir de 1,5 metros presentes nas vias urbanas, sendo observado o nome popular, e utilizando câmera digital SAMSUNG ${ }^{\circledR}$, modelo NV3 para registro fotográfico de alguns espécimes. Para o processo de identificação foi utilizada literatura específica (BRAGA 1978; GRAF 1978; LORENZI, 2002a, LORENZI, 2002b, LORENZI, 2005) além de comparação com material do herbário Arruda Câmara/Centro de Ciências Biológicas e da Saúde/Universidade Estadual da Paraíba. Em seguida os dados foram comparados com os resultados obtidos por Dantas e Souza (2004).

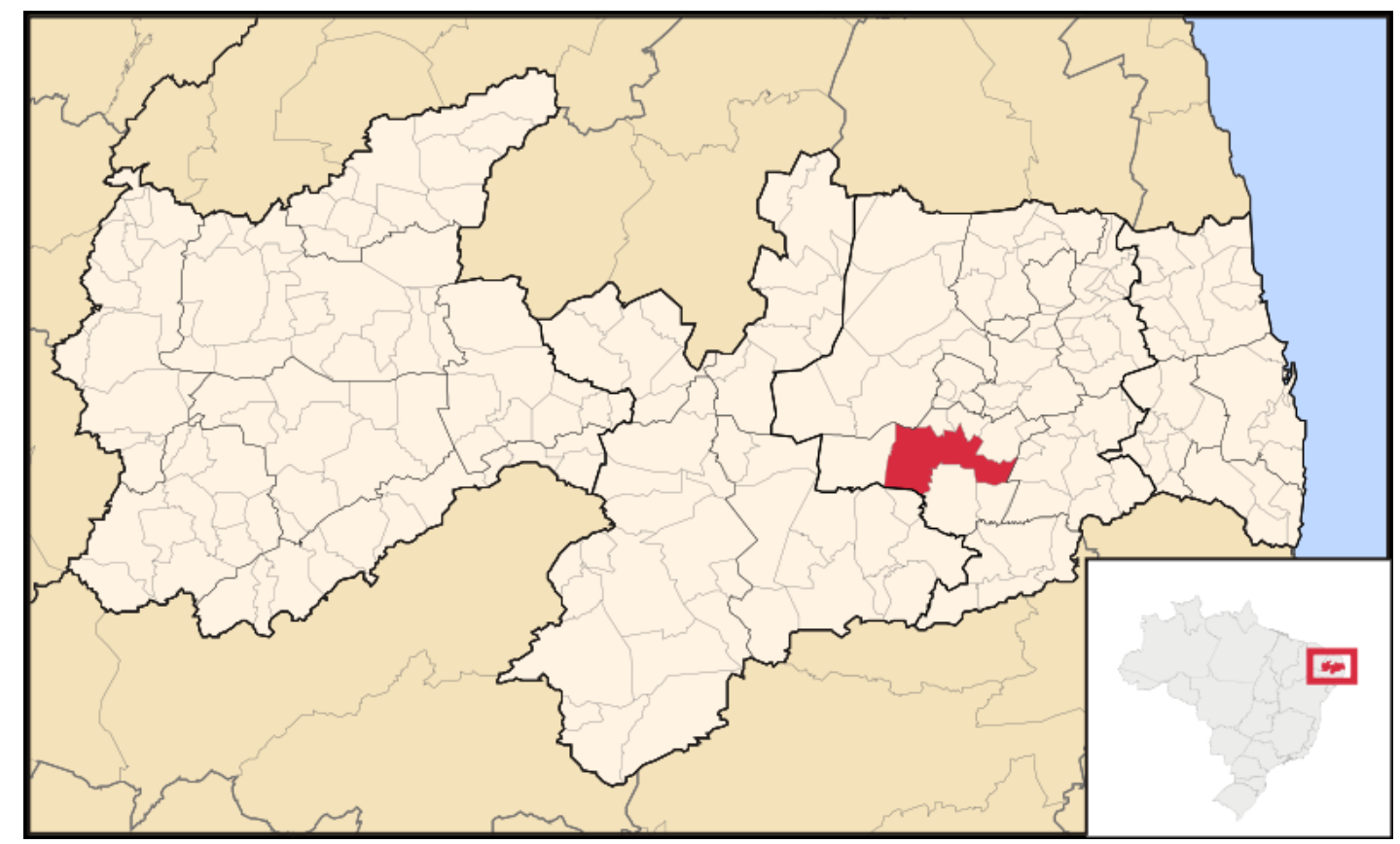

FIGURA 1: Localização do município de Campina Grande-PB.

FIGURE 1: Location of city of Campina Grande, PB.

Fonte: Wikipédia

\section{RESULTADOS E DISCUSSÃO}

$\mathrm{Na}$ arborização urbana de Campina Grande-PB foi realizado por Dantas e Souza (2004) um inventário no período de novembro a 1997 a junho de 1998, que consistiu na contagem das árvores presentes em ruas, avenidas, cemitérios, parques e praças, e teve continuidade no período entre dezembro 1999 e janeiro de 2000 em prédios públicos e privados, sendo incluídos universidades, hospitais, igrejas, shoppings, supermercados, sociedades de bairros e institucionais (Tabela 1).

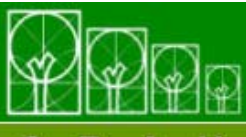

S $\cdot \mathbf{B} \cdot \mathbf{A} \cdot \mathbf{U}$ Soc. Bras. de Arborização Urbana

REVSBAU, Piracicaba - SP, v.6, n.2, p.76-89, 2011 
TABELA 1: Resumo do inventário arbóreo de Campina Grande - PB.

TABLE 1: Summary of tree inventory of Campina Grande, PB.

\begin{tabular}{cc}
\hline Inventário realizado entre 1997-98 & 26.233 \\
Inventário em áreas fechadas 1999-2000 & 2.190 \\
\hline Total & 28.423 \\
\hline
\end{tabular}

Durante o presente estudo, constatou-se que nos Bairros Alto Branco, Lauritzen e Santo Antônio, foram identificadas 3.019 árvores pertencentes a 93 espécies, sendo, as espécies com maior número de exemplares, Ficus (Ficus benjamina L.), Ipê-rosa (Tabebuia pentaphylla Hemsl) e Cássia-amarela Senna siamea Lam (H. S. Irwin \& R. C. Barneby) com 27,89\%, 9,93\% e 8,31\% do total dos espécimes, respectivamente. No inventário concluído em 1998, os referidos bairros apresentavam 1.628 árvores de 56 espécies, com destaque para Cássia-amarela, que representava 22,91\% dos indivíduos (Tabela 1).

O número de espécies encontradas estudo é maior do que o encontrado em outras cidades brasileiras por Volpe-Filik et al. (2007) em Piracicaba - SP (38 espécies), Rossato et al. (2008) em Assis-SP (54 espécies) e Almeida (2009) em cinco cidades do Mato Grosso, as quais apresentaram entre 19 e 8 espécies.

Analisando a evolução da arborização no bairro do Alto Branco, observa-se na tabela 2 o aumento, tanto no número de espécimes como de espécies. No primeiro inventário foram encontradas 858 árvores pertencentes a 44 espécies, enquanto neste, foram registradas 1.698 indivíduos de 73 espécies, representando o aumento de 840 árvores (97,9\%) e 29 espécies (65,9\%). Constatou-se que a espécie de maior destaque em 1998 foi a Senna siamea Lam H. S. Irwin \& R. C. Barneby, com 195 árvores, diminuindo esse número para 157 em 2009. Outro ponto que chama a atenção são as espécies Ficus benjamina L.com um aumento de 20 para 521 espécimes (2.605\%) e Tabebuia sp (variedades rosa, amarela e roxa), as quais apresentaram um aumento de e de 19 para 178 árvores (937\%).

TABELA 2: Listagem das espécies utilizadas na arborização urbana nos bairros do Alto Branco, Lauritzen e Santo Antônio, em Campina Grande - PB. 
TABLE 2: List of species used in urban forest in Alto Branco, Lauritzen e Santo Antônio neighborhoods in Campina Grande, PB.

\begin{tabular}{|c|c|c|c|c|c|c|c|}
\hline \multirow{3}{*}{ Nome popular } & \multirow{3}{*}{ Nome Científico } & \multicolumn{6}{|c|}{ Número de espécimes } \\
\hline & & \multicolumn{2}{|c|}{ Alto Branco } & \multicolumn{2}{|c|}{ Lauritzen } & \multicolumn{2}{|c|}{$\begin{array}{c}\text { Santo } \\
\text { Antônio } \\
\end{array}$} \\
\hline & & $\begin{array}{l}\text { 1997- } \\
98\end{array}$ & 2009 & $\begin{array}{l}\text { 1997- } \\
98\end{array}$ & 2009 & $\begin{array}{l}1997- \\
98\end{array}$ & 2009 \\
\hline Abacateiro & Persea americana Mill. & 1 & 1 & - & - & - & - \\
\hline Abricó-de-macaco & Couroupita guianensis Aubl. & - & - & - & 1 & - & - \\
\hline Acerola & Malpighia glabra L & - & 1 & - & - & - & - \\
\hline Algaroba & Prosopis juliflora DC & 39 & 39 & 7 & 8 & 23 & 26 \\
\hline Algodão-do-pará & Hybiscus pernambucensis Arr.Cam. & 4 & 8 & - & 3 & 2 & 3 \\
\hline Amora & Morus nigra L. & - & 1 & - & - & - & - \\
\hline Araticum & Annona sp & - & 1 & 1 & 1 & - & - \\
\hline Aroeira & Schinus terebinthifolius Raddi & 18 & 55 & 2 & 21 & - & 25 \\
\hline Barriguda & Ceiba pentandra Gaertn & 1 & 4 & - & - & - & - \\
\hline Bouganville & Bougainvillea glabra Choisy & - & 3 & - & 11 & - & 3 \\
\hline Cacau-bravo & Pachira aquatica Aubl & 49 & 40 & 34 & 32 & 16 & 11 \\
\hline Cajazeira & Spondias mombin L. & - & - & - & - & 1 & - \\
\hline Cássia-amarela & $\begin{array}{l}\text { Senna siamea Lam (H. S. Irwin \& R. } \\
\text { C. Barneby) }\end{array}$ & 195 & 157 & 73 & 47 & 105 & 53 \\
\hline Cássia-brasil & Adenanthera pavonina L.. & 18 & 15 & 4 & 6 & 8 & 2 \\
\hline Cássia-grande & Cassia grandis L.f. & - & - & 2 & 11 & 10 & 6 \\
\hline Cássia-fistula & Cassia fistula L. & 2 & 2 & - & 7 & - & 1 \\
\hline Cássia-rosa & Cassia grandis L.f. & 8 & 7 & - & - & - & - \\
\hline Cássia-rósea & Cassia javanica L. & 11 & 17 & 8 & 11 & 3 & 1 \\
\hline Castanhola & Terminalia catappa L. & 97 & 69 & 73 & 51 & 13 & 19 \\
\hline Casuarina & Casuarina equisetifolia L. & 14 & 1 & 11 & 4 & - & - \\
\hline Cedro & Cedrela fissilis Vell & - & 2 & - & - & - & - \\
\hline Chapéu-de-napoleão & $\begin{array}{l}\text { Thevetia peruviana (Pers.) K. } \\
\text { Schum }\end{array}$ & - & 2 & - & - & - & - \\
\hline Cheflera-gigante & $\begin{array}{l}\text { Schefflera actinophylla (Endl) } \\
\text { Harms }\end{array}$ & - & 1 & - & - & - & - \\
\hline Coqueiro & Cocos nucifera L. & 1 & 5 & - & - & - & - \\
\hline Coração-de-negro & Albizzia lebbeck (L.) Benth. & 1 & - & - & 1 & 2 & 1 \\
\hline Craibeira & Tabebuia caraiba (Mart) Bureau & 25 & 39 & - & 10 & 2 & 19 \\
\hline Cupressus & Thuja orientalis L. & 12 & 6 & 7 & 6 & - & - \\
\hline
\end{tabular}

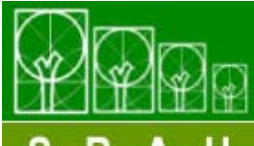

S $\cdot \mathbf{B} \cdot \mathbf{A} \cdot \mathbf{U}$ Soc. Bras. de Arborização Urbana 


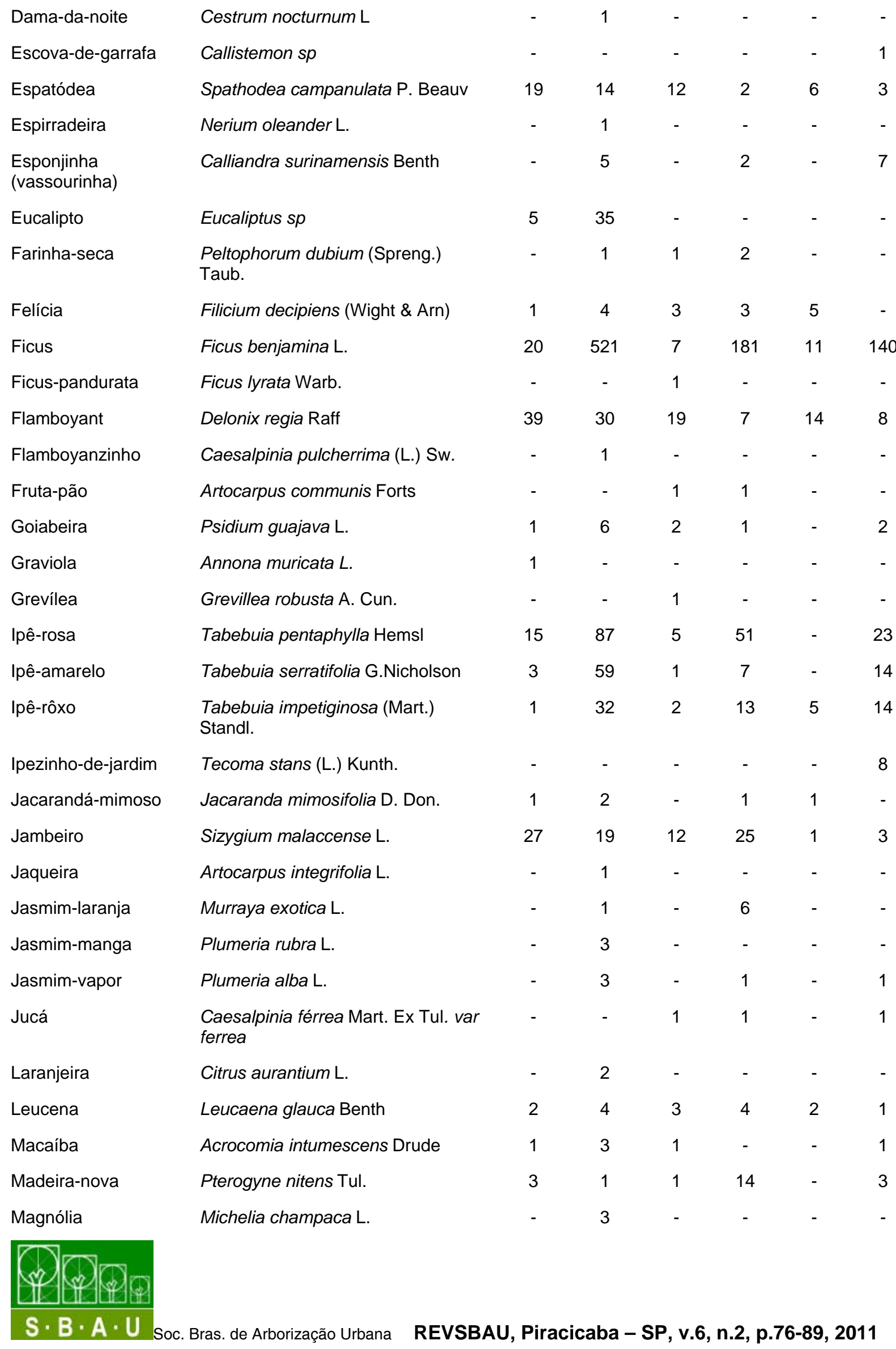




\begin{tabular}{|c|c|c|c|c|c|c|}
\hline Mangueira & Mangifera indica L. & 4 & 9 & 4 & 5 & - \\
\hline Mata-fome & Pithecolobium dulce Benth & 23 & 25 & 43 & 59 & 15 \\
\hline Mororó & Bauhinia monandra Kurz. & 9 & 11 & 5 & 5 & 16 \\
\hline Mulungu & Erythrina velutina Willd. & 1 & 14 & - & - & - \\
\hline Mulungu-indiano & $\begin{array}{l}\text { Erythrina indica Lam, var. picta } \\
\text { Hort, }\end{array}$ & - & 67 & 1 & 13 & - \\
\hline Muringa & Moringa oleifera Lam & - & 1 & - & - & - \\
\hline Não identificada & Não identificada & - & 6 & - & - & - \\
\hline Neem & Azadirachta indica A. Jussieu & - & 37 & - & 3 & - \\
\hline Nogueira & Aleurites moluccana Willd & - & - & 1 & - & - \\
\hline Oiticica & Licania rigida Benth. & 1 & 1 & - & - & - \\
\hline Oitizeiro & Licania tomentosa (Benth) Fritsch. & 10 & 22 & 7 & 11 & 9 \\
\hline Oliveira & Syzygium jambolanum DC. & 14 & 18 & 27 & 80 & 7 \\
\hline Paineira & Sterculia foetida L. & 2 & 3 & - & - & - \\
\hline Palmeira & $\begin{array}{l}\text { Ptychosperma elegans (R. Brown) } \\
\text { Blume }\end{array}$ & 1 & 3 & - & - & - \\
\hline Palmeira-de-jardim & Diypsis lutescens H. Wendl & - & 5 & - & - & - \\
\hline Palmeira-dendê & Elaeis guineensis L. & - & 1 & - & - & - \\
\hline Palmeira-imperial & Roystonea oleracea Cook & - & 13 & - & - & - \\
\hline $\begin{array}{l}\text { Palmeira-rabo-de- } \\
\text { peixe }\end{array}$ & Caryota mitis Lour. & - & 5 & - & - & - \\
\hline Palmeirinha & Dypsis lutescens H. Wendl. & - & 1 & - & - & - \\
\hline Pau-brasil & Caesalpinia echinata Lam. & 7 & 9 & 2 & 5 & 3 \\
\hline Pinha & Annona squamosa L. & - & - & - & 1 & - \\
\hline Pinheiro & Pinus elliottii L. & 2 & 3 & - & - & - \\
\hline Pinheiro araucária & Araucaria columnaris (Forst,) Hook. & - & - & - & 1 & - \\
\hline Pipoca & Lagerstroemia indica var. alba & - & - & - & - & - \\
\hline Pitangueira & Eugenia uniflora L. & - & 3 & - & - & - \\
\hline Pitombeira & Talisia esculenta (A. St.-Hil.) Radlk & - & 1 & - & 2 & - \\
\hline Quaresmeira & Tibouchina sp & - & - & - & - & - \\
\hline Romã & Punica granatum $\mathrm{L}$. & - & - & - & - & 1 \\
\hline Sabonete & Sapindus saponaria L. & 1 & 1 & - & - & - \\
\hline Seriguela & Spondias purpurea L. & - & 1 & - & - & - \\
\hline Sibipiruna & Caesalpinia peltophoroides Benth. & - & - & - & - & 5 \\
\hline Sombreiro & Cliptoria fairchildiana Howard & 145 & 120 & 53 & 33 & 45 \\
\hline
\end{tabular}




\begin{tabular}{|c|c|c|c|c|c|c|c|}
\hline Tamareira & Phoenix sylvestris Roxburg & - & 1 & - & - & - & - \\
\hline Tamarindo & Tamarindus indica Linn & 3 & 1 & - & - & 1 & 1 \\
\hline Tambor & $\begin{array}{l}\text { Enterolobium contortisiliquum(Vell.) } \\
\text { Morong. }\end{array}$ & - & 1 & - & - & - & - \\
\hline & Total & 858 & 1.698 & 438 & 771 & 332 & 559 \\
\hline
\end{tabular}

No bairro Lauritzen também foram observadas várias mudanças quanto à diversidade e ao número de indivíduos, que aumentaram de 35 para 46 espécies (31,42\%) e de 438 para 718 árvores (76\%). As espécies em destaque no inventário anterior foram a Senna siamea Lam (H. S. Irwin \& R. C. Barneby e Terminalia catappa L., as quais apresentavam 73 espécimes cada uma, no ano de 2009, verificou-se redução das referidas espécies para 47 e 51 árvores, respectivamente. No presente inventário destaca-se o Ficus benjamina L. que passou de 7 indivíduos no inventário de 1997-98 para 181 em 2009, o que representa um aumento de 2.585\%. Também merecem destaque Tabebuia sp (rosa, amarelo e roxo), os quais passaram de 8 para 71 espécimes, equivalendo a um aumento de 887,5\% (Tabela 2).

Com relação ao bairro Santo Antônio, observa-se no tabela 2 que esse bairro apresenta na arborização de suas 20 ruas, 559 árvores, contra 332 existentes em 1998, representando um aumento de 168,4\%, bem como o acréscimo no número de espécies, que passou de 28 para 45 (37,7\%). Observa-se que, a espécie mais representativa no bairro no ano de 1998 foi a Senna siamea Lam (H. S. Irwin \& R. C. Barneby com 105 árvores, em 2009, detectou-se decréscimo expressivo no número de árvores da referida espécie, o qual foi reduzido quase a metade. Quanto às espécies mais representativas, em 2009, são Ficus benjamina L. que teve um aumento de $1.272,7 \%$.

Apesar de todos os bairros estudados apresentarem um aumento no número de espécies e de espécimes, a espécie Ficus benjamina L. apresentou um crescimento muito acentuado, passando de 38 para 842 indivíduos, o que representa 27,89\% das árvores existentes. O acréscimo dessa espécie em Campina Grande pode ter se ocasionado pelo modismo já que essa espécie exótica se adaptou bem ao clima local, apresentando crescimento rápido e folhagem brilhante o ano inteiro, fatores que conquistaram adeptos.

De acordo com Lorenzi et al. (2003), Ficus benjamina L. é comum na arborização de calçadas e parques, no entanto devido às suas raízes superficiais e bem desenvolvidas, essa espécie não é adequada para o plantio em calçadas. Além disso, na literatura há relatos que esta espécie é prejudicial à estrutura viária da cidade, sendo responsável por (4) $\theta 0$

$\mathbf{S} \cdot \mathbf{B} \cdot \mathbf{A} \cdot \mathbf{U}$ Soc. Bras. de Arborização Urbana 
danos à calçadas, calçamento, bem como conflitos com as redes aéreas. Santana e Santos (1999) observaram indivíduos do gênero Ficus utilizados na arborização no Campus da Universidade Estadual de Feira de Santana (UEFS) danificando calçadas, Paula e Melo (2010) constataram que Ficus benjamina L. foi uma das espécies com maior número de indivíduos causando danos nas calçadas no município de Planalto-SP.

Outra espécie que mereceu destaque foi Ipê-rosa (Tabebuia pentaphylla Hemsl) a qual apresentou um acréscimo de 805\%, passando de 20 exemplares para 161 árvores. Essa espécie mesmo sendo exótica é indicada por Dantas et al (2010) como apropriadas a serem utilizadas na arborização de cidades em praça, parques, ruas, quintais, estacionamentos, jardins e em reflorestamento.

Rocha et al., (2004) ressaltam que a predominância de apenas uma espécie ou grupo de espécies pode promover a difusão de pragas, cada vez mais comuns nas árvores urbanas, podendo uma única praga e/ou doença comprometer toda a arborização.

O fato de poucas espécies representarem um grande número de indivíduos também é comum em outras cidades brasileiras como Jaboticabal-SP (SILVA FILHO, 2002), Águas de São Pedro-SP (BORTOLETO et al. 2007), Aracaju-SE (LIMA NETO et al., 2007), Pombal-PB (RODOLFO JÚNIOR et al., 2008), Lavras da Mangabeira-CE (CALIXTO JÚNIOR et al., 2009) e Rio Branco-AC (PAIVA et al., 2010).

\section{CONCLUSÕES}

De acordo com o estudo realizado conclui-se que:

Apesar do número de espécies e de espécimes dos bairros Alto Branco, Lauritzen e Santo Antônio terem aumentado consideravelmente, ainda é insuficiente.

Ocorreu um acréscimo bastante acentuado de indivíduos da espécie Ficus benjamina L. que apresenta atualmente $27,89 \%$ das árvores contabilizadas. Esse crescimento é preocupante, pois uma praga pode comprometer boa parte da arborização dos referidos bairros.

Esses resultados mostram a necessidade de um melhor planejamento da arborização da cidade, ampliando ainda mais o número de árvores, dando ênfase para as espécies nativas e acentuando a heterogeneidade florística. 
É necessário ainda, o desenvolvimento de programa de educação ambiental, visando sensibilizar a população sobre a importância da arborização urbana, como também preservá-la de maneira adequada e eficiente, aperfeiçoando seu efeito paisagístico; orientar a população sobre a maneira adequada do plantio e manutenção, garantindo a redução da depredação e supressão de árvores.

\section{REFERÊNCIAS BIBLIOGRÁFICAS}

ALMEIDA, D. N. Análise da arborização urbana de cinco cidades da região norte do estado de Mato Grosso. 2009. 62 f. Dissertação (Mestrado em Ciências Florestais e Ambientais) Universidade Federal de Mato Grosso, Cuiabá - MT.

BALENSIEFER, M.; WIECHETECK, M. Arborização das cidades. Curitiba: ITCF/Secretaria Estadual da Agricultura e Abastecimento, 1987.

BORTOLETO, S. Inventário quali-quantitativo da arborização viária da estância de Águas de São Pedro-SP. 98p. Dissertação (Mestrado) - Escola Superior de Agricultura "Luiz de Queiroz", Universidade de São Paulo, Piracicaba, 2004.

BRAGA, R. Plantas do Nordeste: especialmente do Ceará. 3. ed. Ceará: Ed. Mossoroense, 1978.

CALIXTO JÚNIOR, J. T.; SANTANA, G. M.; LIRA FILHO, J. A. Análise quantitativa da arborização urbana de Lavras da Mangabeira, CE, Nordeste do Brasil. Revista da Sociedade Brasileira de Arborização Urbana, Piracicaba - SP, v.4, n.3, p.99-109, 2009.

COLTRO, E. M.; MIRANDA, G. M. Levantamento da arborização urbana pública de Irati - PR e sua influência na qualidade de vida de seus habitantes. Revista Eletrônica Lato Sensu, v. 2, no 1, jul., p. 1-22, 2007.

CRHISTO, J.A; DIAS, A.N. Inventário Florestal da Arborização Urbana do Centro da Cidade de Prudentópolis - PR. Revista Eletrônica Lato Sensu. Ano 2, no1, p. 76-93. 2007.

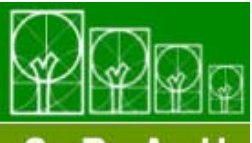

$\mathbf{S} \cdot \mathbf{B} \cdot \mathbf{A} \cdot \mathbf{U}$ Soc. Bras. de Arborização Urbana

REVSBAU, Piracicaba - SP, v.6, n.2, p.76-89, 2011 
DANTAS, I.C; SOUZA, C.M.C. de. Arborização urbana na cidade de Campina Grande - PB: Inventário e suas espécies. Revista e Biologia e Ciências da Terra. Volume 4 - Número 2 $2^{\circ}$ Semestre 2004.

DANTAS, I. C. et al. Manual de Arborização Urbana. Campina Grande: Eduepb, 2010.

FALEIRO, W.; AMÂNCIO-PEREIRA, F. Arborização viária do campus Umuarama da Universidade Federal de Uberlândia, MG. Revista Científica Eletrônica de Engenharia Florestal. vol. 6, n. 10, ago. 2007.

GRAF, A. B. Tropica. East Rutherford. Roehrs Company, 1978.

KULCHETSCKI, L. et al. Arborização urbana com essências nativas: uma proposta para a Região Centro-Sul brasileira. Publ. UEPG Ci. Exatas Terra, Ci. Agr. Eng., Ponta Grossa, 12 (3): 25-32, dez. 2006.

LIMA NETO, E. M. et al. Análise das áreas verdes das praças do bairro Centro e principais avenidas da cidade de Aracaju - SE. Revista da Sociedade Brasileira de Arborização Urbana, V. 2, n. 1, p. 17-33, 2007.

LORENZI, H. Árvores brasileiras: manual de identificação e cultivo de plantas arbóreas do Brasil. 4.ed. Nova Odessa: Instituto Plantarum, 2002a. v.1, 384p.

LORENZI, H. Árvores brasileiras: manual de identificação e cultivo de plantas arbóreas do Brasil. 2.ed. Nova Odessa: Instituto Plantarum, 2002b. v.2, 384p.

LORENZI, H. et al. Árvores exóticas no Brasil: madeiras, ornamentais e aromáticas. Nova Odessa: Instituto Plantarum, 2003. v.1, 368p.

MASCARENHAS, J. de C.; et al. (Orgs.) Projeto cadastro de fontes de abastecimento por água subterrânea. Diagnóstico do município de Campina Grande. Recife: CPRM/PRODEEM, 2005. 
MELO, R. R.; LIRA FILHO, J. A.; RODOLFO JÚNIOR, F. Diagnóstico qualitativo e quantitativo da arborização urbana no bairro Bivar Olinto, Patos, Paraíba. Revista da Sociedade Brasileira de Arborização Urbana, v. 2, n. 1, p. 1-17, 2007.

MILANO, M. S. Curso de Manejo de Áreas Silvestres. Curitiba: FUPEF, 1983.

PAIVA, A. V. et al. Inventário e diagnóstico da arborização urbana viária de Rio Branco, AC. Revista da Sociedade Brasileira de Arborização Urbana. Piracicaba - SP, v.5, n.1, p.144-159, 2010.

PAULA, D. de S.; MELO, A. G. C. de. Levantamento quali-quantitativo da arborização urbana do município de Planalto, SP. Revista Científica Eletrônica de Engenharia Florestal., v.16, n.1, ago, 2010.

PEDROSA, J.B. Arborização de cidades e rodovias. Belo Horizonte -MG: E.F. 1983.

ROCHA, R. T.; LELES, P. S. dos S.; OLIVEIRA NETO, S. N. de. Arborização de vias públicas em Nova Iguaçu, RJ: o caso dos bairros Rancho Novo e Centro. R. Árvore, ViçosaMG, v.28, n.4, p.599-607, 2004.

RODOLFO JÚNIOR, F. et al. Análise da arborização urbana em bairros da cidade de Pombal no estado da Paraíba. Revista da Sociedade Brasileira de Arborização Urbana, Piracicaba - SP, v.3, n.4, p.3-19, 2008.

ROSSATO, D. R.; TSUBOY, M. S. F.; FREI, F. Arborização urbana na cidade de Assis/SP: uma abordagem quantitativa. Revista da Sociedade Brasileira de Arborização Urbana, v. 3, n. 3, set., p. 1-16, 2008.

SANTANA, J.R.F.; SANTOS, G.M.M. Arborização do campus da UEFS: exemplo a ser seguido ou um grande equívoco? Sitientibus, n.20, p.103-107, 1999. 
SOUZA, M. C. de. Arborização urbana: inventário e suas espécies. 2001. 44 p. Monografia (Graduação) Universidade Estadual da Paraíba, Campina Grande. 2001.

VOLPE-FILIK, A.; SILVA, L. F.; LIMA, A. L. M. P.; Avaliação da arborização de ruas do bairro São Dimas nas cidade de Piracicaba/SP através de parâmetros qualitativos. Revista da Sociedade Brasileira de Arborização Urbana, Piracicaba, v. 2, n. 1, mar., p. 34-43, 2007. 